\title{
PAPER
}

\section{The genetic jigsaw of inflammatory bowel disease}

\section{A Watts, J Satsangi}

Gut 2002;50(Suppl III):iii31-iii36

Following a prolonged period of relative inertia, real progress has been made in the past few years in understanding the pathogenesis of the chronic inflammatory bowel diseases, Crohn's disease and ulcerative colitis. Clinical experience, epidemiological studies, and molecular genetics have provided strong evidence that both genetic and environmental factors are important in disease pathogenesis, and gene-environmental interaction determines disease susceptibility and behaviour.

See end of article for authors' affiliations

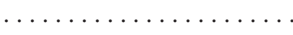

Correspondence to: Dr D A Watts, Gastrointestinal Unit, The University of Edinburgh, Western General Hospital, Edinburgh EH4 2XU, UK; dwatts@ed.ac.uk
$T$ he chronic inflammatory bowel diseases (IBD), Crohn's disease and ulcerative colitis are common causes of gastrointestinal morbidity in Western Europe and Northern America. Recent population based studies suggest that the combined prevalence of these diseases in the United Kingdom approaches 400 per $100000^{1}$; complementary studies show that the incidence of Crohn's disease in young people in the United Kingdom has continued to rise in recent years. ${ }^{2}{ }^{3}$ Although mortality from these diseases is low, the associated problems of chronic ill health, hospital admission, drug toxicity, and surgery present an important source of morbidity, particularly among young people. The effects of these illnesses on growth, education, career development, and social wellbeing may be profound.

Following a prolonged period of relative inertia, real progress has been made in recent years in understanding the genesis of Crohn's disease and ulcerative colitis. Epidemiological data have provided incontrovertible evidence that both genetic and environmental factors are important in both disease susceptibility, and also as determinants of disease progression. It has become apparent that gene-environmental interactions underlie disease development and progression. The use of genetically engineered animals, which become prone to chronic intestinal inflammation, has emphasised the central role of immune dysregulation in disease pathogenesis and also the contribution of the enteric flora. ${ }^{4}$

However, studies of human genetics have provided the greatest insight into disease pathogenesis. The complementary techniques of genome wide scanning and candidate gene analysis have provided strong evidence that the chronic inflammatory bowel diseases are related polygenic disorders, sharing some but not all the susceptibility loci. The successful application of genome wide scanning has contrasted with inconsistent data in other complex disorders. A number of replicated regions of linkage have been identified, culminating with the identification of the IBD 1 gene on chromosome 16 as Nod 2. The data implicating this gene in Crohn's disease are compelling, and appear to provide strong evidence for a primary defect in the innate immune response to bacteria in inflammatory bowel disease. The recent papers from independent investigators in France and North America represent a landmark in complex disease genetics. ${ }^{56}$

There is real optimism that rapid progress will follow and give rise to early clinical application. These fundamental advances in our understanding of disease pathophysiology may lead to advances in patient counselling, patient stratification for therapy, and in the development of novel non-toxic therapies.

\section{EVIDENCE FOR GENETIC SUSCEPTIBILITY IN INFLAMMATORY BOWEL DISEASE}

There is strong epidemiological evidence to suggest that genetic factors are important in the pathogenesis of Crohn's disease and ulcerative colitis. The most compelling data are those from the three recent studies of concordance rates in twin pairs in Europe..$^{7-9}$ These data supplement a number of studies carried out in Europe and the United States which show a high prevalence of familial inflammatory bowel disease in relatives of patients with Crohn's disease and ulcerative colitis. $^{10}$

The study by Tysk et al of the Swedish registry of twin births ${ }^{7}$ catalysed the epidemiological, clinical, and subsequently molecular genetic studies into inflammatory bowel disease genetics, which have taken place since its publication in 1988. The data reported by the Swedish group have been closely replicated by the investigators in Great Britain" and Denmark. ${ }^{8}$ In total 322 twin pairs have now been reported. Combining the data from the European studies allows concordance rates in Crohn's disease of $37 \%$ in monozygotic twin pairs, and $7 \%$ in dizygotic twin pairs to be derived. In ulcerative colitis the equivalent result in monozygotic and dizygotic twins were respectively $10 \%$ and $3 \%$.

From the concordance rate reported, the coefficient of heritability (the relative contribution of inherent susceptibility to disease pathogenesis) may be derived. This figure is particularly noteworthy in Crohn's disease, where the heritability is at least equivalent to values derived in insulin dependent diabetes and multiple sclerosis.

Abbreviations: IBD, inflammatory bowel disease; LPS, lipopolysaccharide; $M H C$, major histocompatibility complex; TNF $\alpha$, tumour necrosis factor $\alpha$; TPMT, thiopurine methyltransferase 
The results of the twin studies have been complemented by the results of studies in familial inflammatory bowel disease. It is now well documented that a positive family history of inflammatory bowel disease is the best established risk factor for development of disease. The precise estimates of the familial risk do vary, but consistent themes are present throughout the studies carried out in Europe and North America. Between $6 \%$ and $32 \%$ of patients with inflammatory bowel disease have an affected first or second degree relative. The relative most commonly affected is the sibling of a patient with inflammatory bowel disease. Parents, offspring, and second degree relatives appear to have a lower risk. Calculation of the relative risk to siblings, $\lambda \mathrm{s}$, has been particularly instructive in inflammatory bowel disease, and has allowed comparison with other complex disorders. The relative risk to a sibling $(\lambda s)$ of a patient with Crohn's disease has been estimated as between 13 and 36, and for ulcerative colitis as between 7 and 17 . Equivalent figures in type 1 diabetes, schizophrenia, and cystic fibrosis, are 15, 8.6, and 400 respectively. ${ }^{10}{ }^{11}$

Moreover, considerable data exist to support genetic determination of not only susceptibility to disease but also disease phenotype. Epidemiological studies in multiply affected kindreds in the United Kingdom have shown high concordance rates for disease type, location, and the presence of extra intestinal manifestations. ${ }^{12}$ Data from the United States sugggest that disease behaviour in Crohn's disease (stenosing, fistulising, or mixed) may also have a familial basis. ${ }^{13}$

On evaluating the data from North America, it is apparent that the risk of familial disease is particularly increased among Jewish patients, and also among patients with an early onset of inflammatory bowel disease. These observations, made independently by investigators at the Cleveland clinic, ${ }^{14}$ and Los Angeles, ${ }^{15}$ and elsewhere, ${ }^{16}$ have been supplemented by molecular genetic data, which suggest that the prospects for gene identification are enhanced in these subgroups of patients.

There are a number of other lines of evidence, which complement the concordance rates in twin pairs and multiply affected families, in providing evidence for genetic susceptibility in inflammatory bowel disease. Association between the inflammatory bowel diseases and other diseases with recognised genetic susceptibility, notably primary sclerosing cholangitis ${ }^{17}$ and ankylosing spondylitis add to this evidence, as do the association with Turner's syndrome. ${ }^{18}$

\section{GENETIC MODEL}

Although the epidemiological data provide strong evidence that genetic factors are important in disease pathogenesis, it is also apparent that environmental factors are critical. The interaction between susceptibility genes and environmental stimuli is likely to determine disease susceptibility and subsequently disease progression. Although complex segregation analyses have suggested that a simple recessive model may be pertinent to a small proportion of patients with Crohn's disease, and a simple dominant model be pertinent to a proportion of patients with ulcerative colitis, ${ }^{19}$ it is likely that the genetic model behind the inflammatory bowel diseases is more complex, and must also account for the variability of clinical presentation, and the co-occurrence of both Crohn's disease and ulcerative colitis in multiply affected families. The model which is most widely accepted at the current time is that Crohn's disease and ulcerative colitis are related polygenic disorders, sharing some but not all susceptibility loci. The disease phenotype is likely to be determined by the interaction between different allelic variants in a number of genes, and the environment. The twin data indicate that the environmental contribution is likely to be relatively stronger in ulcerative colitis than Crohn's disease. This complex model has received support from not only the epidemiological data currently available, but also from the results of molecular genetic studies—both candidate gene approaches, and also the results of genome wide scanning.

\section{SEARCHING FOR GENES IN COMPLEX DISEASES}

In recent years, huge investments of scientific and financial resources have been made, in the attempt to identify genetic determinants important in common complex diseases. Although studies in inflammatory bowel disease began somewhat later than those in insulin dependent diabetes, asthma, or common psychiatric diseases, the rate of progress in identifying genetic determinants in Crohn's disease and ulcerative colitis has been striking among all complex diseases. The complementary strategies of genome wide scanning and candidate gene analysis have identified determinants of disease susceptibility, progression, and response to drug therapy. In particular, the identification of the IBDl gene by independent investigators in France ${ }^{5}$ and North America ${ }^{6}$ represents, without exaggeration, an important scientific breakthrough and is likely to be viewed as such for many years. In discovering this gene, the authors made use of the results of previous linkage analysis studies involving affected sibling pairs, fine mapping studies involving families with one affected member, and the wealth of data available "in silico" as a consequence of the human genome mapping project.

\section{GENOME WIDE SCANNING IN INFLAMMATORY BOWEL DISEASE}

Weissenbach and colleagues ${ }^{20}$ were first able to report the development of a linkage map of the human genome, involving microsatellite markers spanning $90 \%$ of the genome. The markers involved were polymorphic, and informative in linkage studies, in tracing inheritance in multiply affected families. The availability of this linkage map, together with subsequent advances in molecular laboratory techniques, notably the semiautomation of genotyping facilities for polymorphic markers, allowed many investigators to search the genome for regions which may contain susceptibility genes important in complex diseases. Initial data were reported in insulin dependent diabetes in 1994, ${ }^{21}$ followed by results for many other complex diseases, together with monogenic disorders.

As experience with the technique accumulated, a true understanding of the requirements for successful genome wide scanning, and of the limitations of the technique have become available. ${ }^{22}$ Many modifications and improvements have been carried out in recent years-not only in molecular genotyping, but also in statistical analysis, and data interpretation. Of particular note is the need for access to large numbers of well phenotyped families, and the need for rigorous data interpretation.

Inflammatory bowel disease has now reached a unique, and enviable position, compared with other complex diseases. A number of replicated regions of linkage have been described, which satisfy even the most stringent criteria imposed by statistical geneticists. The two regions of linkage initially described in $1996,{ }^{24}{ }^{25}$ on chromosomes 16 and 12 have, in particular, been subjected to rigorous reanalysis throughout the world. Most impressive however, is the success in fine mapping and gene identification for the IBDI locus.

\section{IBD1-FROM LINKAGE TO GENE}

Hugot and colleagues, ${ }^{24}$ based in Paris, reported the first genome screen in Crohn's disease in 1996. The investigators initially genotyped 40 affected sibling pairs in 25 French families using 270 markers throughout the genome. Analysis of allele sharing in affected sibling pairs identified only four markers achieving a p value of 0.01 or less. Of these, three were on chromosome 1, and one on chromosome 16. However, in a smaller second set of families, supportive evidence for linkage 
with the region on chromosome 16 only was obtained. The authors designated the region of linkage on chromosome 16 as IBDI, and estimated the sibling ratio specific to this locus as 1.3 .

There were worries regarding the validity of this localisation, at the time of the original publication. A relatively small number of families have been studied, and the effect of the gene appeared to be relatively modest.

However, the experience in the subsequent five years has entirely validated the original publication, and has provided an important lesson in the genetics of complex diseases. The linkage in Crohn's disease has been almost universally replicated throughout the world. ${ }^{96}$ In addition, the International IBD Genetics Consortium involving 12 groups of investigators has pooled data concerning 603 affected sibling pairs, and provided incontrovertible evidence for replication of the chromosome 16 linkage. ${ }^{26}$ Hugot and colleagues, moreover, have proceeded from the initial description of linkage to gene identification. Refinement of the localisation of the IBDI susceptibility locus was carried out by genotyping 26 microsatellite markers spaced at an average distance of 1 centimorgan in the pericentromeric region of chromosome 16. The investigators then constructed a physical map of the region using contiguous bacterial artificial chromosomes. The authors pursued this classical positional cloning strategy after showing association between susceptibility to Crohn's disease and a specific microsatellite marker. The authors used a complementary series of polymorphic markers, single nucleotide polymorphisms, within the physical region of interest to further fine map the region of interest, and identified a single gene within which the single nucleotide polymorphisms showed very strong evidence for association with Crohn's disease. The gene thus identified encodes Nod 2, a member of the Apaf-1 super family of apoptosis regulators, expressed in monocytes. A number of polymorphisms within the Nod 2 gene were shown to be associated with Crohn's disease susceptibility, of which the strongest association lay with a single nucleotide insertion in exon 10. The insertion of a cytosine repeat in exon 10 gives rise to a truncated form of the Nod 2 gene. Of particular note is the fact that this allelic variant affects a leucine repeat rich sequence of the gene, which has strong homology with leucine rich repeat regions of the Toll like receptor gene super family. The Toll like receptors represent an integral part of the innate immune response to bacterial infection, and the leucine rich repeat sequence encodes the region of the molecule which interacts with bacterial antigens.

Hugot and colleagues' data suggest that the Nod 2 gene has a recessive mode of action. The relative risk for simple heterozygotes is estimated as 3, whereas the risk for simple homozygotes is 38 , and the relative risk compared with the general population for compound heterozygotes (carrying a combination of polymorphisms of the gene) is quoted at 44 .

Hugot and colleagues' report implicating this gene is based on a positional cloning strategy, and has identified a plausible candidate gene. The evidence implicating the Nod 2 gene is further supplemented by the simultaneous publication ${ }^{6}$ from investigators from the United States. These authors analysed the Nod 2 gene, as a plausible positional candidate gene within the linkage interval on chromosome 16, and concentrated their attentions on the same insertion of a cytosine at nucleotide 3020. As Hugot and colleagues had reported, this insertion results in a frameshift mutation at the second nucleotide of codon on 1007 , leading to a premature stop codon, and protein truncation. Using both case control strategies, and an intrafamilial association study (relying on transmission disequilibrium), the authors were able to show strong evidence for association with Crohn's disease. In the case control study, the allelic frequencies of the 3020 ins $C$ were comparable among Jewish (8.4\%) and non-Jewish $(8.1 \%)$ patients with Crohn's disease, contrasted with the control allelic frequency $(4 \%)$. These authors calculated genotype relative risk for heterozygous and homozygous individuals, and again showed the likely recessive affect of the 3020 insC mutation. In fact the genotype relative risk for heterozygotes was only 1.5, emphasising the need to identify other genetic and non-genetic determinants of disease.

Ogura and colleagues ${ }^{6}$ also investigated the functional effect of the 3020 ins $C$ mutation, by assessing the ability of wild type and mutant Nod 2 to activate NF- $\mathrm{KB}$ in human embryonic kidney cell lines. Although equivalent levels of wild type and mutant resulted in similar levels of NF- $\mathrm{KB}$ activation in the absence of bacterial lipopolysaccharide (LPS), striking differences were noted in the functional effect of wild type and mutant genes in the presence of bacterial LPS. Somewhat counter intuitively, the mutant Nod 2 was capable of reduced NF- $\kappa \mathrm{B}$ activation after LPS stimulation, compared with wild type.

These data have produced real excitement, not only in inflammatory bowel disease, but also in other complex disorders. However, a number of important questions now result, involving not only the functional effect of the mutation described, but also interaction with other genetic factors, and with environmental determinants. This discovery is likely to have wide reaching effects in complex diseases, and to stimulate a further re-evaluation of the contribution of the innate immune system in many immune mediated diseases.

\section{THE IBD2 LOCUS}

Shortly after Hugot and colleagues' initial report, Satsangi and colleagues reported evidence for further susceptibility loci in both Crohn's disease and ulcerative colitis, ${ }^{25}$ having studied 186 affected sibling pairs from 160 UK families. The strongest evidence for linkage was found for a region spanning 41 centimorgans on the long arm of chromosome 12. Five adjacent microsatellite markers within this region showed distortion of allele sharing, with the most significant evidence for linkage being seen at the marker D12S83 (in the initial dataset, lod score $5.47, \mathrm{p}=2.7 \times 10^{-7}$ ). Suggestive evidence for linkage with inflammatory bowel disease was also seen on chromosome 7 (three adjacent markers, of which the strongest evidence for linkage was achieved with D7S669) and chromosome 3 (two adjacent markers, lod score 2.69). These regions were implicated in both Crohn's disease and ulcerative colitis; in addition this initial UK dataset provided weak evidence for linkage in ulcerative colitis alone (regions on chromosome 6 and 2) and in Crohn's disease alone (IBD1). Although the chromosome 12 linkage has been strongly replicated in a number of datasets, ${ }^{27}{ }^{28}$ replication has not been universal. ${ }^{29}$ Indeed, the Inflammatory Bowel Disease Consortium Study found maximum lod scores for inflammatory bowel disease, ulcerative colitis, and Crohn's disease of only 1.8, 1.2, and 1.1 respectively. ${ }^{26}$ There are many possible explanations for the lack of widespread replication-and it is likely that a combination of factors underlies the current data. However, a collaborative study between the investigators in Oxford and Pittsburgh has identified the most plausible explanation for the discrepancies. Parkes and colleagues ${ }^{30}$ argue that the strongest evidence for linkage with the IBD2 locus has in fact been seen in datasets in which there are a significant number of ulcerative colitis families. In contrast, weak or no evidence for linkage has been noted in datasets in which there are few, or no ulcerative colitis pedigrees. Analysis of the combined Oxford/Pittsburgh data have shown heterogeneity at the IBD2 locus between ulcerative colitis (lod score 3.91) and Crohn's disease (lod score 1.66). Thus these investigators argue that the IBD2 gene may be purely involved in the pathogenesis of ulcerative colitis. Although gene identification has not yet been successfully carried out, fine mapping of this region in ulcerative colitis dominated datasets is likely to be fruitful in the near future, given the sequence information now available, and the physical map which has already been 
constructed of this region. ${ }^{31}$ A number of plausible candidate genes have been investigated with negative results, including the beta 7 integrin gene, ${ }^{32}$ and the STAT- 6 gene. ${ }^{33}$

\section{IBD3-CONTRIBUTION OF THE MAJOR HISTOCOMPATIBILITY COMPLEX}

The genes of the major histocompatibility complex (MHC) have received considerable attention in genetic studies in inflammatory bowel disease. The recent increased understanding of the complexity of inflammatory bowel disease-in particular the extent of disease heterogeneity-has helped explain the inconsistent results of earlier studies. It is now well accepted that the genes of the MHC influence not only disease susceptibility but also disease behaviour in inflammatory bowel disease. The relative contribution of the HLA region, compared with other genetic determinants has been estimated in a number of studies. Data from Europe, ${ }^{34}{ }^{35}$ based on the allelic sharing pattern in affected siblings and reported allelic associations, suggest that the contribution of the HLA region in ulcerative colitis is stronger than that in Crohn's disease. However, genome wide scanning ${ }^{36}$ and candidate gene studies $^{37}$ in Crohn's disease suggest that the HLA region may carry up to a third of the genetic contribution to disease susceptibility.

\section{WHICH GENES IN THE HLA REGION ARE INVOLVED PRIMARILY IN DISEASE SUSCEPTIBILITY?}

Although well designed studies are currently underway to answer this question, at present it remains unclear whether the primary contribution of the HLA region reflects the effect of class I or class II molecules, or any of the other hundreds of genes within this region, many of which are involved in immunoregulation. The genes of the major histocompatibility complex are marked by considerable sequence polymorphism, ${ }^{38}$ which has been attributed to the evolutionary effect of host-bacterial antagonism. Although allelic association with HLA class I, class II, and class III genes are now well described, there continues to be considerable uncertainty as to whether the allelic associations simply reflect the effect of the pronounced linkage disequilibrium within the region. Nevertheless, a number of striking associations have been described in recent years. Although overall association with disease susceptibility is relatively weak, in white Europeans, strong associations between disease behaviour and HLA allelic variation have been described, particularly in ulcerative colitis. It has become apparent that the HLADR B ${ }^{*} 0103$ allele may predict severity of ulcerative colitis, need for surgery, and the presence of extraintestinal manifestations. ${ }^{39}$ Striking associations have been described with arthropathy, other extraintestinal manifestations, and disease extent. ${ }^{40}$ Although these remain to be tested prospectively in clinical practice, there is strong accumulated evidence, which would support the clinical use of these markers. It is noteworthy that ethnic differences in the contribution of the HLA region are very apparent. In Japanese patients, ulcerative colitis is associated strongly with HLA DRB1*1502, an allele rarely seen in the white, non-Jewish population. ${ }^{34}$

Of the number of genes within the HLA class III region, the genes encoding tumour necrosis factor $\alpha(\mathrm{TNF} \alpha)$ require particular mention. Increasing clinical evidence supports the pivotal role of TNF $\alpha$ in the development and progression of Crohn's disease. In both Japanese ${ }^{42}$ and European ${ }^{43}$ populations, allelic variation within the promoter region of the TNF $\alpha$ gene has been associated with susceptibility to and progression of Crohn's disease. Most recent data strongly implicate this region in susceptibility to Crohn's disease, and it is of particular interest as to whether genetic determinants may predict response to monoclonal antibody therapy directed against TNF $\alpha$. The data at the present time are inconsistent. $^{4445}$

\section{CHROMOSOME 14-THE IBD4 LOCUS}

Increasing evidence implicates the subchromosomal region on chromosome 14q11-12 as being involved in susceptibility to Crohn's disease. Four groups ${ }^{296-48}$ of investigators have reported evidence of linkage to this region, with varying degrees of statistical significance. All of the datasets studied have been dominated by Crohn's disease families-notably these studies from Pittsburgh, Los Angeles, Belgium, and Chicago. All investigators have used the complementary strategies of linkage analysis and association study to attempt narrowing the region of linkage on chromosome 14, and unpublished preliminary data do suggest that the region may be narrowed by these analyses.

\section{OTHER LOCI-TRUE OR FALSE: SUGGESTIVE OR SIGNIFICANT?}

A number of other regions have been implicated in nine genome scans which have now been reported throughout the world. Since the initial studies reported in 1996, methodologies for genotyping analysis have become evermore sophisticated, and it is indeed likely that many linkages reported worldwide do represent true susceptibility loci, awaiting adequate replication studies. Thus, although the International IBD Genetics Consortium continues to concentrate on IBD $1-4$, there are ample grounds to justify detailed studies of the other loci detected in Australian, ${ }^{49}$ US, Canadian, ${ }^{50}$ and European populations. Indeed, as our understanding of the genetic determinants present in these regions increases, the relative contributions of each of the loci in individual populations is likely to become more apparent. The interaction between individual genetic determinants is also likely to be critical, and early data from the large North American genome scan published by Cho and colleagues ${ }^{29}$ amply illustrates this. The investigators were able to identify novel loci on chromosomes $1 \mathrm{p}, 3 \mathrm{q}$, and $4 \mathrm{q}$, and also suggest an epistatic interaction between the locus on chromosome $1 \mathrm{p}$ and the IBDl gene.

Since the initial genome wide scans, the challenge for investigators has been the progression from description of linkage to gene identification. The recent success for the chromosome 16 locus is therefore all the more important. The progression from genome scan to precision mapping to physical mapping of the gene in IBDl provides an example for investigators involved in identifying the other loci of importance, not only in inflammatory bowel disease but also other complex polygenic diseases.

\section{FROM BENCH TO BEDSIDE?}

Clinicians and patients are now eagerly awaiting the translation from basic research to clinical application. Although considerable progress has been made, the data at present are not sufficiently robust to allow gene testing for inflammatory bowel disease, and there are a number of ethical difficulties which will further confound progress in this direction. The genes which have been and are likely to be identified will only denote predisposition towards inflammatory bowel disease, and it will need to be carefully pointed out that gene-gene, and gene-environmental interaction will be critical in the development of disease.

The development of novel, disease specific therapies for inflammatory bowel disease has remained the goal of many investigators involved in inflammatory bowel disease genetics research. With the identification of the Nod 2 gene, this goal seems ever more realistic. The development of new drugs, whose mode of action is based on the increased understanding of disease pathophysiology, is now becoming a realistic and tangible proposition, which may justify the huge investment in complex disease genetics. 
In inflammatory bowel disease, it is hoped that the first clinical application may be in the field of pharmacogeneticsusing genetic markers to predict responsiveness, or tolerance of available therapeutic agents. Already a number of publications suggest that response to azathioprine, anti-TNF $\alpha$ agents, and corticosteroids may have a genetic basis.

Metabolism of azathioprine and 6-mercaptopurine is complex. Azathioprine is non-enzymatically converted to 6-mercaptopurine, which in turn is either inactivated by TPMT or xanthine oxidase, or activated through a number of enzymatic steps to the putative active metabolites, the 6-thioguanine nucleotides. Attention has focused on the activity of TPMT. ${ }^{51}$ The TPMT gene is polymorphic and a large number of variant alleles have been described, having reduced enzymatic activity. There has been considerable interest in relating efficacy or side effects of azathioprine to TPMT activity and genotype. Although not yet in widespread use, it does seem increasingly likely that a combination of TPMT activity, and 6-thioguanine nucleotide concentrations may be used in monitoring efficacy and toxicity in the near future. However, Colombel and colleagues have pointed out that the toxicity of azathioprine reflects not only TPMT activity, but a number of other environmental, as well as likely genetic factors. ${ }^{52}$

The use of anti-TNF $\alpha$ agents, notably infliximab, the chimeric monoclonal antibody, has been the major advance in IBD therapeutics over the past few years. The efficacy of this drug in otherwise medically refractory patients has been noteworthy. However, infliximab itself is only effective in a proportion of patients with Crohn's disease; there has been considerable interest in using subclinical markers to determine drug responsiveness and to stratify patients for therapy. As yet, data are preliminary, and bear replication studiespolymorphisms of the lymphotoxin gene, ${ }^{53}$ TNF $\alpha$ gene, ${ }^{44} 45$ TNF receptor 2 gene, and Nod 2 gene $^{56}$ have been studied. However, further data are eagerly awaited, particularly in view of the considerable expense of this and other newer biological therapies.

Many intriguing data suggest molecular markers which may help predict responsiveness to corticosteroids in inflammatory bowel disease. The most well replicated association is with the HLADRB ${ }^{*} 0103$ allele in resistant ulcerative colitis ${ }^{39}$; however, other data are also reported for the IאBL gene within the HLA class III region, and other determinants. ${ }^{54}$ In view of the clinical importance of steroid resistance in inflammatory bowel disease, this area of research may well translate to clinical application in the near future.

Success in gene identification has been eagerly awaited by investigators, clinicians, and patients involved with inflammatory bowel disease. Real progress has undoubtedly been made in recent years in the laboratory, and there is now great optimism that this progress will give rise to early, tangible clinical benefit.

\section{SUMMARY}

Following a prolonged period of relative inertia, real progress has been made in the past few years in understanding the pathogenesis of the chronic inflammatory bowel diseases, Crohn's disease and ulcerative colitis. Clinical experience, epidemiological studies, and molecular genetics have provided strong evidence that both genetic and environmental factors are important in disease pathogenesis, and geneenvironmental interaction determines disease susceptibility and behaviour.

Genome wide scanning in inflammatory bowel disease has now been carried out successfully by investigators throughout the world. Four regions of linkage have been widely replicated: IBDI on chromosome 16 (implicated in Crohn's disease only), IBD2 (chromosome 12q13), IBD3 (the major histocompatibility complex on chromosome 6p23), and chromosome 14q11-12 (IBD4). The extent of replication of these loci, particularly the IBDl locus, is remarkable, when compared with inconsistent results shown in other complex diseases. However, fine mapping of the regions of linkage to identify the susceptibility genes themselves has provided a formidable challenge. The study of large numbers of families of given ethnicity and phenotype has been undertaken as an international collaborative venture, in the attempt to narrow linkage intervals.

Most recently, data from the United States and France both suggest that the IBDI gene has been identified, a considerable breakthrough in IBD genetics. The Nod 2 gene, involved in $\mathrm{NF \kappa B}$ activation, is implicated by these studies and the polymorphisms associated with Crohn's disease focus interest on the innate immune response to enteric flora.

The current challenge is to translate the scientific progress of recent years into real clinical benefit. There is considerable optimism that prediction of drug tolerability and efficacy will result from recent pharmacogenetic studies-in particular studies of the contribution of polymorphism of the TPMT (thiopurine methyltransferase) gene in azathioprine/6mercaptopurine metabolism is nearing clinical application. In addition genetic markers may predict the efficacy and tolerability of new biological therapies (anti-TNF $\alpha$ antibody) and corticosteroid drugs. The development of new specific therapies for inflammatory bowel disease may become reality within the next decade, on the basis of the recent exciting progress.

...............

Authors' affiliations

D A Watts, J Satsangi, Gastrointestinal Unit, The University of Edinburgh, Western General Hospital, Edinburgh, UK

\section{REFERENCES}

1 Rubin GP, Hungin AP, Kelly PJ, Ling J. Inflammatory bowel disease: epidemiology and management in an English general practice population. Aliment Pharmacol Ther 2000;14:1561-5.

2 Armitage EA, Drummond H, Ghosh S, Ferguson A. Incidence of juvenile onset Crohn's disease in Scotland. Lancet 1999;353:1496-7.

3 Sawczenko C, Sandhu BK, Logan RFA, et al. Prospective survey of childhood inflammatory bowel disease in the British Isles. Lancet 2001;357:1093-4.

4 Sellon RK, Tonkonogy S, Schultz M, et al. Resident enteric bacteria are necessary for development of spontaneous colitis and immune system activation in interleukin 10 deficient mice Infect. Infect Immun 1998;66:5224-31.

5 Hugot J-P, Chamaillard M, Zonali $\mathrm{H}$, et al. Association of NOD-2 leucine rich repeat variants with susceptibility to Crohn's disease. Nature 2001;411:599-603.

6 Ogura Y, Bonen DK, Inohara N, et al. A frameshift mutation in NOD2 associated with susceptibility to Crohn's disease. Nature $2001 ; 411: 603-6$

7 Tysk C, Lindberg E, Järnerot G, Flodérus-Myrhed B. Ulcerative colitis and Crohn's disease in an unselected population of monozygotic and dizygotic twins. A study of heritability and the influence of smoking. Gut 1988;29:990-6.

8 Orholm M, Binder V, Sorensen T, Kyvik K. Inflammatory bowel disease in a Danish twin register. Gut 1996;39(suppl 3):A187.

9 Thompson NP, Driscoll R, Pounder RE, Wakefield AJ. Genetics versus environment in inflammatory bowel disease: results of a British twin study. BM 1996;312:95-6.

10 Ahmad T, Satsangi J, McGovern D, et al. Review article: the genetics of inflammatory bowel disease. Aliment Pharmacol Ther 2001;15:731-8.

11 Satsangi J, Jewell DP, Bell Jl. Leading article. The genetics of inflammatory bowel disease. Gut 1997;40:572-4

12 Satsangi J, Grootscholten C, Holt H, Jewell DP. Clinical patterns of familial inflammatory bowel disease. Gut 1996;38:738-41.

13 Bayless TM, Tokayer AZ, Polito JM, et al. Crohn's disease: concordance for site and clinical type in affected family members - potential hereditary influences. Gastroenterology 1996;111:573-9.

14 Farmer RG, Michener WM, Mortimer EA. Studies of family history among patients with inflammatory bowel disease. Clin Gastroenterol 1980;9:271-7.

15 Yang $\mathbf{H}$, McElree C, Roth M-P, et al. Familial empirical risks for inflammatory bowel disease: differences between Jews and non-Jews. Gut 1993;34:517-24.

16 Silver J. Unpublished observations.

17 Chapman RWL. Aetiology and natural history of primary sclerosing cholangitis: a decade of progress? Gut 1991;32:1433-5.

18 Hayward PA, Satsangi J, Jewell DP. Inflammatory bowel disease and the X chromosome. QJM 1996;89:713-18. 
19 Orholm M, Iselius L, Sorensen TI, et al. Investigation of inheritance of chronic inflammatory bowel diseases by complex segregation analysis. BM 1993;306:20-4

20 Weissenbach J, Gyapay G, Dib C, et al. A second generation linkage map of the human genome. Nature 1992;359:794-801.

21 Hashimoto L, Habita C, Beressi JP. Genetic mapping of a susceptibility locus for insulin-dependent diabetes mellitus on chromosome $11 \mathrm{q}$. Nature 1994;371:161-4.

22 Kruglyak $L$, Lander $E$. Limits on fine mapping of complex traits [letter]. Am J Hum Genet 1996;58:1092-3.

23 Risch N. Searching for genetic determinants in the new millennium. Nature 2000;405:847-56.

24 Hugot JP, Laurent-Puig P, Gower-Rousseau C, et al. Mapping of a susceptibility locus for Crohn's disease on chromosome 16. Nature 1996:379:821-2.

25 Satsangi J, Parkes $M$, Lovis $E$, et al. Two-stage genome-wide search in inflammatory bowel disease provides evidence for susceptibility loci on chromosomes 3, 7 and 12. Nat Genet 1996;14:199-202.

26 The IBD Consortium, Cavanaugh J. The International IBD genetics consortium confirms linkage of Crohn's disease to a locus on chromosome 16 (IBD1). Gastroenterology 2000;118:A3862.

27 Duerr RH, Barmada MM, Zhang L, et al. Linkage and association between inflammatory bowel disease and a locus on chromosome 12 Am J Hum Genet 1998;63:95-100.

28 Curran ME, Lau KF, Hampe J, et al. Genetic analysis of inflammatory bowel disease in a large European cohort supports linkage to chromosomes 12 and 16. Gastroenterology 1998;115:1066-71.

29 Cho JH, Nicolae DL, Gold LH, et al. Identification of novel susceptibility loci for inflammatory bowel disease on chromosomes $1 \mathrm{p}, 3 \mathrm{q}$, and $4 \mathrm{q}$ : evidence for epistasis between $1 \mathrm{p}$ and IBDI. Proc Natl Acad Sci 1998;95:7502-7.

30 Parkes M, Barmada M, Satsangi J, et al. Linkage heterogenetity between ulcerative colitis and Crohn's disease at the IBD2 locus. Am J Hum Genet 2000;67:1605-10.

31 Hamlin P, Komolmit P, Bransfield K, et al. Identification of multiple candidate genes for IBD susceptibility using high density transcript mapping in the IBD2 locus on chromosome 12q. Gastroenterology 1999; 117:1029-31

32 Van Heel D, Jewell DP, Carey AH. Novel polymorphisms in the beta 7 integrin gene family based association studies in IBD [abstract A470]. Gastroenterology 2001;(suppl):2325

33 Dimon C, Allen M, Van Heel D, et al. Family based association studies of STAT6, a positional candidate gene for IBD [abstract A471] Gastroenterology 2001;(suppl):2333.

34 Satsangi J, Welsh KI, Bunce $M$, et al. Contribution of genes of the major histocompatibility complex to susceptibility and disease phenotype in inflammatory bowel disease. Lancet 1996;347:1212-17.

35 Stokkers PC, Reitsma PH, Tytgat GN, van Deventer SJ. HLA-DR and -DQ phenotypes in inflammatory bowel disease: a meta-analysis. Gut 1999:45:395-401.

36 Hampe J, Schreiber S, Shaw S, et al. A genome-wide analysis provides evidence for novel linkages in inflammatory bowel disease in a large European cohort. Am J Hum Genet 1999;64:808-16.
37 Yang H, Plevy S, Taylor K. Linkage of Crohn's disease to the major histocompatibility complex region is detected by multiple non-parametric analyses. Gut 1999;44:519-26.

38 The MHC sequencing consortium. Complete sequence and gene map of a major histocompatibility complex. Nature 1999;401:921-3.

39 Roussomoustakaki M, Satsangi J, Welsh K, et al. Genetic markers may predict disease behaviour in patients with ulcerative colitis. Gastroenterology 1997;112:1845-53

40 Orchard T, Thiyagaraja S, Welsh K, et al. Clinical phenotype is related to HLA genotype in the peripheral arthropathies of inflammatory bowel disease. Gastroenterology 2000;1 18:274-8.

41 Sugimura K, Asakura H, Mizuki N, et al. Analysis of genes within the $\mathrm{HLA}$ region affecting susceptibility to ulcerative colitis. Hum Immunol 1993;36:112-18

42 Negoro K, Kinouchi $Y$, Hiwatashi N, et al. Crohn's disease is associated with novel polymorphisms in the $5^{\prime}$ flanking region of the tumour necrosis gene. Gastroenterology 1999;117:1062-8.

43 Kinouchi Y, Van Heel D, Cardon L, et al. Transmission dysequilibrium testing confirms the association of the TNF $\alpha 1031 \mathrm{C}$ allele with Crohn's disease [abstract A469]. Gastroenterology 2001;(suppl):2321.

44 Vermeire S, Monsuu F, Groenen P, et al. Response to anti TNFa treatment is associated with the TNFa-308* 1 allele [abstract]. Gastroenterology 2000;1 18(suppl 2):A3595

45 Marion J, Bodian C, Lisa T, et al. TNF microsatellite polymorphism does not predict response to infliximab in patients with Crohn's disease. Gastroenterology 2000;118(suppl 2).

$46 \mathrm{Ma} \mathrm{Y}$, Ohmen J, Li Z, et al. A genome wide search identifies potentia new susceptibility loci for Crohn's disease. Inflamm Bowel Dis 1999;5:271-8

47 Duerr R, Barmada M, Zhang L, et al. High-density genome scan in Crohn's disease shows confirmed linkage to chromosome 14q11-12. Am J Hum Genet 2000;66:1857-62.

48 Vermeire S, Vlietinck R, Groenen P, et al. Replication of linkage on $14 q 11-12$ in inflammatory bowel disease. Gastroenterology 2000;118(suppl 2):A338.

49 Cavanaugh JA. Unpublished data.

50 Rioux JD, Silverberg MS, Daly M, et al. Am J Hum Genet 2000;66:1863-70

51 Cuffari C, Hunt S, Bayless E. Utilisation of erythrocyte-thioguanine metabolite levels to optimise azathioprine therapy in patients with inflammatory bowel disease. Gut $2001 ; 48: 642-6$.

52 Colombel JF, Ferrari N, Debuysere H, et al. Genotypic analysis of thiopurine 5-methyltransferase in patients with Crohn's disease and severe myelosuppression during azathioprine therapy. Gastroenterology 2000; 1 18: 1025-30.

53 Taylor KD, Plevy SE, Yang H, et al. ANCA pattern and LTA haplotype relationship to clinical responses to anti-TNF antibody treatment in Crohn's disease. Gastroenterology 2001;120:1347-55.

54 De la Concha EG, Fernandex-Arquero M, Lopez-Nava G, et al. Susceptibility to severe ulcerative colitis is associated with polymorphism in the central MHC gene, IKBL. Gastroenterology 2000;1 19:1491-5. 